\title{
Charge Balancing Fill Rate Monitor
}

\author{
J.L. Rothman and E.B. Blum \\ National Synchrotron Light Source, Brookhaven National Laboratory, Upton, New York 11973
}

\section{Abstract}

A fill rate monitor has been developed for the NSLS storage rings to allow machine tuning over a very large dynamic range of beam current. Synchrotron light, focused on a photodiode, produces a signal proportional to the beam current. A charge balancing circuit processes the diode current, creating an output signal proportional to the current injected into the ring. The unit operates linearly over a dynamic range of $120 \mathrm{~dB}$ and can resolve pulses of injected beam as small as $1 \mu \mathrm{A}$.

\section{INTRODUCTION}

The fill rate monitor is one of the most frequently used diagnostic tools in the NSLS control room, allowing the operator to optimize injection and reduce the time between fills. Previous fill rate monitors have used signals from pickup electrodes (PUEs) or from a DC current transformer (DCCT). A tuned receiver connected to a PUE can measure very small currents, but tends to saturate with large signals. The DCCT tolerates large signals but is too noisy to resolve small changes in current. The new unit combines the best elements of the other systems, providing both high sensitivity and a wide dynamic range.

\section{DESIGN}

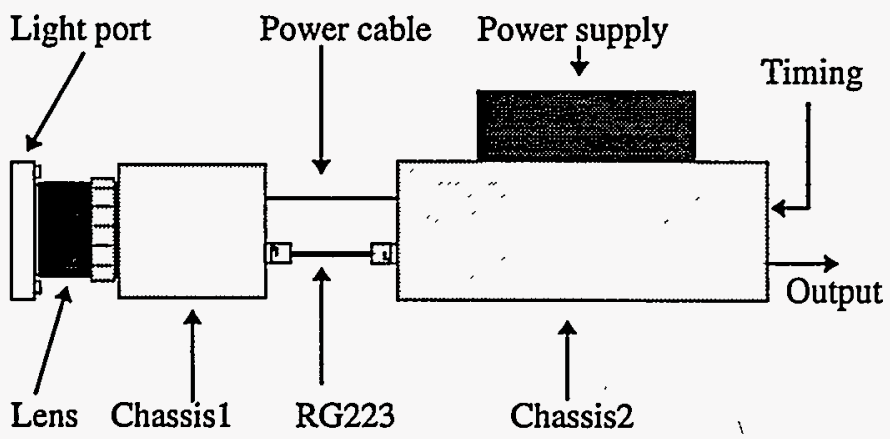

Figure 1: Schematic view of the mechanical design

The mechanical design is shown in figure 1 . The lens is a standard video camera lens mounted on the light port via an adapter. It focuses the light into chassis 1 and onto the photodiode. The diode is mounted on a micrometer so the

* Work performed under the auspices of the U.S. Department of Energy beam spot can be centered. Current from the diode runs through a double shielded cable to the electronics located in chassis 2 .

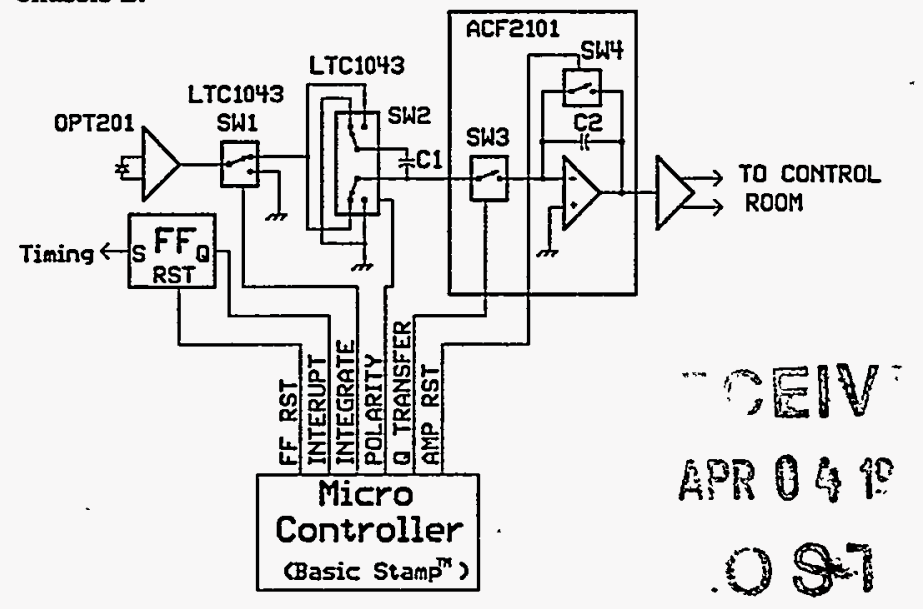

Figure 2: Electronic System diagram

ALL SIGNALS ACTIUE LOW

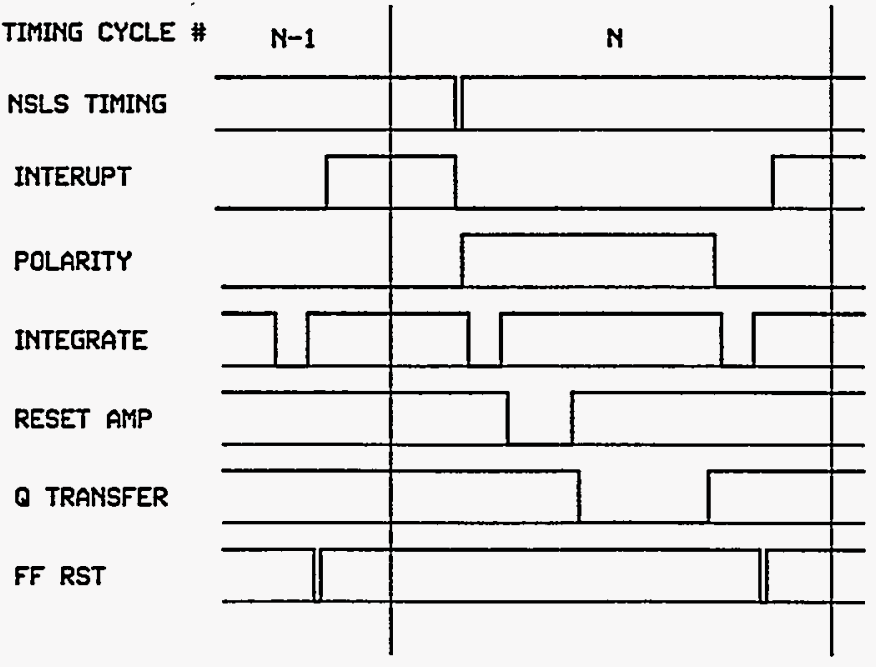

Figure 3: Timing diagram

The electronic system and timing diagrams are shown in figure 2 and figure 3 respectively. At the end of timing cycle $\mathrm{N}-1$, the absolute current in the ring is measured by closing SW1 and integrating the photodiode current on the bottom plate of $\mathrm{C} 1$ for $50 \mathrm{mS}$. The interrupt flip flop is then reset in preparation for timing cycle N. A timing pulse sets the flip flop and interrupts the microcontroller $100 \mathrm{mS}$ after a shot has been injected into the ring. This allows the beam to damp and

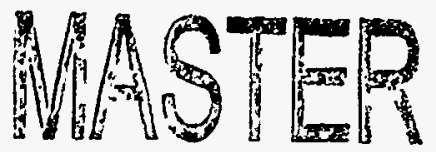




\section{DISCLAIMER}

This report was prepared as an account of work sponsored by an agency of the United States Government. Neither the United States Government nor any agency thereof, nor any of their employees, make any warranty, express or implied, or assumes any legal liability or responsibility for the accuracy, completeness, or usefulness of any information, apparatus, product, or process disclosed, or represents that its use would not infringe privately owned rights. Reference herein to any specific commercial product, process, or service by trade name, trademark, manufacturer, or otherwise does not necessarily constitute or imply its endorsement, recommendation, or favoring by the United States Government or any agency thereof. The views and opinions of authors expressed herein do not necessarily state or reflect those of the United States Government or any agency thereof. 


\section{DISCLAIMIER}

Portions of this document may be illegible in electronic image products. Images are produced from the best available original document. 
prevents fluctuations in the diode current due to beam motion. The microcontroller then reverses the polarity of SW2. SW1 closes again and integrates the photodiode current on the top plate of $\mathrm{Cl}$. The charge remaining on $\mathrm{Cl}$ is proportional to the current injected into the ring. At this point SW4 closes, resetting the charge sensitive amplifier. After SW4 opens SW3 closes to transfer the charge from $\mathrm{Cl}$ to $\mathrm{C2}$, updating the output signal. Since $\mathrm{C} 1=10 \mu \mathrm{F}$ and $\mathrm{C} 2=4.7 \mathrm{nF}$ the voltage gain $=2100$. The output is buffered with a differential driver and transmitted to the control room for display on a digital oscilloscope.

The OPT201[1] is a low noise photodiode and a transimpedance amplifier fabricated on the same chip. In this application it has been configured to provide a current output. SW1 and SW2 are LTC1043 [2] FET switches. C1 is made large to keep the input signals near ground. This makes the switch charge injection less than $1 \mathrm{pC}$, contributing less than $200 \mu \mathrm{V}$ to the output offset voltage. The ACF2101 [1] is a dual analog integrator also with low noise and low charge injection characteristics.

A Basic Stamp ${ }^{\mathrm{TM}}$ [3] microcontroller sequences the FET switches. Since the Stamp is programmed in a dialect of the Basic programming language, only 11 executable lines of code are required to produce the needed timing signals. Programs are downloaded from a PC via the parallel port.

Variables can be sent back to the PC at run time for debugging purposes, making an emulator unnecessary. The code is stored in an EEPROM so programs can be updated at any time.

\section{PERFORMANCE}

The resolution as a function of beam current is shown in figure 4 . The noise floor is given by:

$$
I_{N}=1 \mu A *\left(1+\left(I_{\text {BEAM }} \mu \mathrm{A} / 27369\right)\right)
$$

Despite the increase in noise at high currents the monitor can still resolve $37 \mathrm{ppm}$ changes in beam current with $850 \mathrm{~mA}$ in the ring.

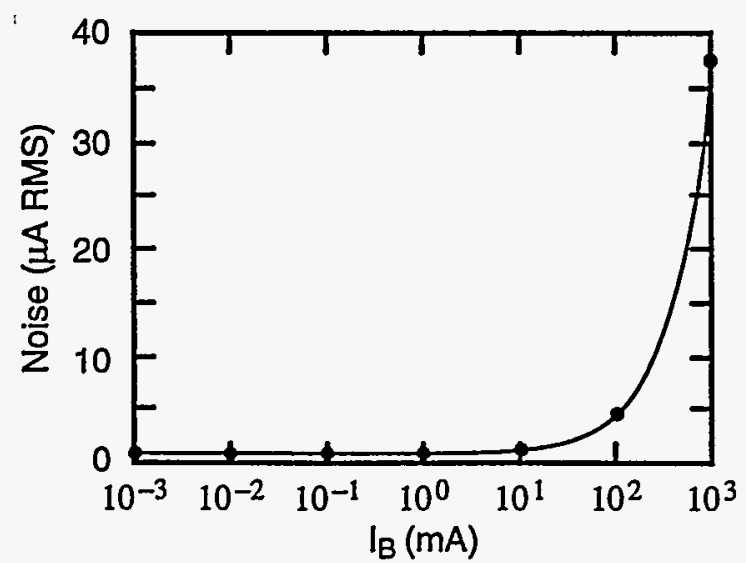

Figure 4: Resolution as a function of beam current

\section{CONCLUSIONS}

The charge balancing technique used in this fill rate monitor allows high resolution measurements to be made over a wide range of beam current. The performance can be further enhanced by moving the unit to a beam port that sees less beam motion. Even so, it measures small changes in current much better than previous detectors, opening the possibility of using charge balancing techniques to measure beam lifetime.

\section{REFERENCES}

[1] Burr Brown Corporation, Linear Products, P.O. Box 11400 Tucson, AZ 85734-1400

[2] Linear Technology Corporation, 1630 McCarthy Blvd, Milpitas, CA 95035

[3] Parallax, Inc. 3805 Atherton Road, \#102, Rocklin, CA 95765

\section{ACKNOWLEDGMENTS}

Thanks to Henry Link for technical support. 


\title{
Charge Balancing Fill Rate Monitor
}

\author{
J.L. Rothman and E.B. Blum \\ National Synchrotron Light Source, Brookhaven National Laboratory, Upton, New York 11973
}

\section{Abstract}

A fill rate monitor has been developed for the NSLS storage rings to allow machine tuning over a very large dynamic range of beam current. Synchrotron light, focused on a photodiode, produces a signal proportional to the beam current. A charge balancing circuit processes the diode current, creating an output signal proportional to the current injected into the ring. The unit operates linearly over a dynamic range of $120 \mathrm{~dB}$ and can resolve pulses of injected beam as small as $1 \mu \mathrm{A}$.

\section{INTRODUCTION}

The fill rate monitor is one of the most frequently used diagnostic tools in the NSLS control room, allowing the operator to optimize injection and reduce the time between fills. Previous fill rate monitors have used signals from pickup electrodes (PUEs) or from a DC current transformer (DCCT). A tuned receiver connected to a PUE can measure very small currents, but tends to saturate with large signals. The DCCT tolerates large signals but is too noisy to resolve small changes in current. The new unit combines the best elements of the other systems, providing both high sensitivity and a wide dynamic range.

\section{DESIGN}

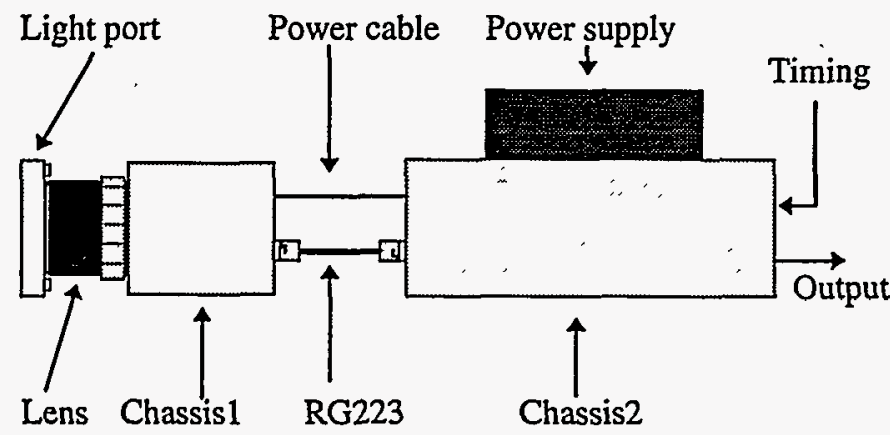

Figure 1: Schematic view of the mechanical design

The mechanical design is shown in figure 1 . The lens is a standard video camera lens mounted on the light port via an adapter. It focuses the light into chassis 1 and onto the photodiode. The diode is mounted on a micrometer so the

* Work performed under the auspices of the U.S. Department of Energy beam spot can be centered. Current from the diode runs through a double shielded cable to the electronics located in chassis 2 .

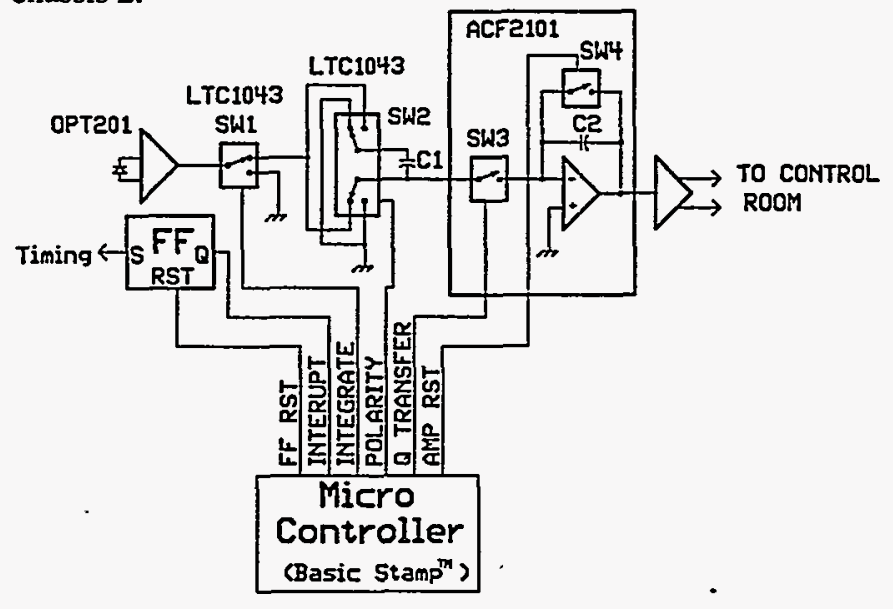

Figure 2: Electronic System diagram

ALL SIGNALS ACTIUE LOW

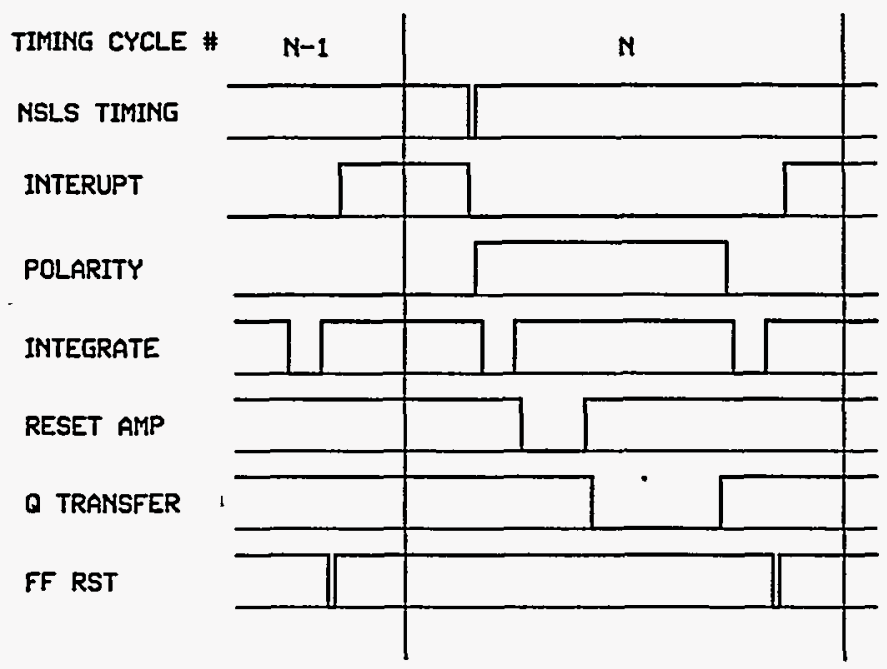

Figure 3: Timing diagram

The electronic system and timing diagrams are shown in figure 2 and figure 3 respectively. At the end of timing cycle $\mathrm{N}-1$, the absolute current in the ring is measured by closing SW1 and integrating the photodiode current on the bottom plate of $\mathrm{C} 1$ for $50 \mathrm{mS}$. The interrupt flip flop is then reset in preparation for timing cycle $\mathrm{N}$. A timing pulse sets the flip flop and interrupts the microcontroller $100 \mathrm{mS}$ after a shot has been injected into the ring. This allows the beam to damp and 
prevents fluctuations in the diode current due to beam motion. The microcontroller then reverses the polarity of SW2. SW1 closes again and integrates the photodiode current on the top plate of $\mathrm{Cl}$. The charge remaining on $\mathrm{C} 1$ is proportional to the current injected into the ring. At this point SW4 closes, resetting the charge sensitive amplifier. After SW4 opens SW3 closes to transfer the charge from $\mathrm{Cl}$ to $\mathrm{C} 2$, updating the output signal. Since $\mathrm{C} 1=10 \mu \mathrm{F}$ and $\mathrm{C} 2=4.7 \mathrm{nF}$ the voltage gain $=2100$. The output is buffered with a differential driver and transmitted to the control room for display on a digital oscilloscope.

The OPT201[1] is a low noise photodiode and a transimpedance amplifier fabricated on the same chip. In this application it has been configured to provide a current output. SW1 and SW2 are LTC1043 [2] FET switches. C1 is made large to keep the input signals near ground. This makes the switch charge injection less than $1 \mathrm{pC}$, contributing less than $200 \mu \mathrm{V}$ to the output offset voltage. The ACF2101 [1] is a dual analog integrator also with low noise and low charge injection characteristics.

A Basic Stamp ${ }^{\mathrm{TM}}$ [3] microcontroller sequences the FET switches. Since the Stamp is programmed in a dialect of the Basic programming language, only 11 executable lines of code are required to produce the needed timing signals. Programs are downloaded from a PC via the parallel port.

Variables can be sent back to the PC at run time for debugging purposes, making an emulator unnecessary. The code is stored in an EEPROM so programs can be updated at any time.

\section{PERFORMANCE}

The resolution as a function of beam current is shown in figure 4 . The noise floor is given by:

$$
I_{N}=1 \mu A *\left(1+\left(I_{B E A M} \mu A / 27369\right)\right)
$$

Despite the increase in noise at high currents the monitor can still resolve $37 \mathrm{ppm}$ changes in beam current with $850 \mathrm{~mA}$ in the ring.

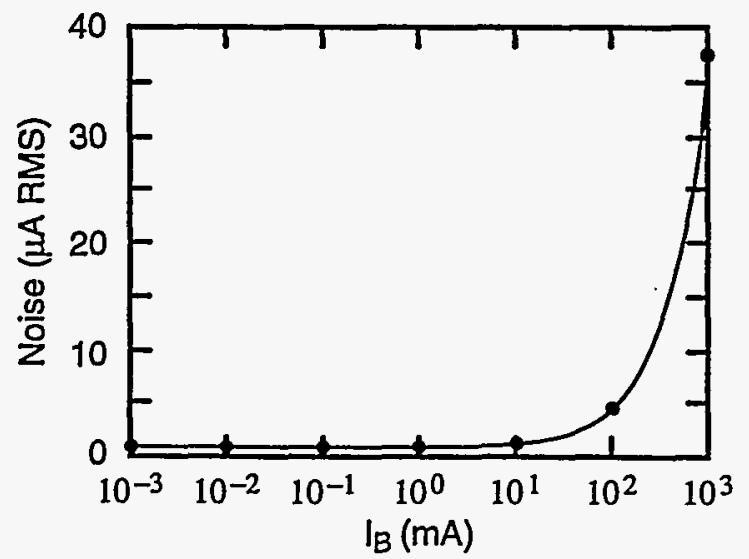

Figure 4: Resolution as a function of beam current

\section{CONCLUSIONS}

The charge balancing technique used in this fill rate monitor allows high resolution measurements to be made over a wide range of beam current. The performance can be further enhanced by moving the unit to a beam port that sees less beam motion. Even so, it measures small changes in current much better than previous detectors, opening the possibility of using charge balancing techniques to measure beam lifetime.

\section{REFERENCES}

[1] Burr Brown Corporation, Linear Products, P.O. Box 11400 Tucson, AZ 85734-1400

[2] Linear Technology Corporation, 1630 McCarthy Blvd, Milpitas, CA 95035

[3] Parallax, Inc. 3805 Atherton Road, \#102, Rocklin, CA 95765

\section{ACKNOWLEDGMENTS}

Thanks to Henry Link for technical support. 